\title{
GRADIENT REALIZATION OF NONLINEAR CONTROL SYSTEMS
}

\author{
J. Cortés * A. J. van der Schaft ${ }^{* *}$ P. E. Crouch ${ }^{* * *}$ \\ * Coordinated Science Laboratory, University of Illinois at \\ Urbana-Champaign, 1308 W. Main St., Urbana, IL 61801, \\ United States, Tel: +1-217-2448734, Fax: +1-217-2441653, \\ Email: jcortes@uiuc.edu \\ ** Department of Applied Mathematics, Systems, Signals \\ and Control group, University of Twente, PO Box 217, \\ 7500 AE Enschede, The Netherlands, Tel: +31 53 \\ 489-3449, Fax: +3153434-0733, \\ Email: a.j.vanderschaft@math.utwente.nl \\ *** Department of Electrical and Computer Engineering, \\ Arizona State University, Tempe, AZ 85287, United \\ States, Tel: +1480 965-1722, Fax: +1480 965-2267, \\ Email: peter.crouch@asu.edu
}

\begin{abstract}
We investigate necessary and sufficient conditions under which a nonlinear affine control system with outputs can be written as a gradient control system corresponding to some pseudo-Riemannian metric defined on the state space. The results rely on a suitable notion of compatibility of the system with respect to a given affine connection, and on the input-output behavior of the prolonged system and the gradient extension. The symmetric product associated with an affine connection plays a key role in the discussion.
\end{abstract}

Keywords: gradient control systems, symmetric product, prolongation and gradient extension

\section{INTRODUCTION}

A physically motivated class of nonlinear systems are gradient systems, see (Crouch 1981, van der Schaft 1984a, van der Schaft 1984b) and the references quoted therein. These are systems endowed with a (pseudo-) Riemannian metric on the state space manifold in such a way that the drift vector field is a gradient vector field with respect to this Riemannian metric and an internal potential function, and the input vector fields are gradient vector fields with potential functions given by the output functions of the system. Examples of gradient systems include nonlinear electrical RLC networks (in the case of RL or RC networks the Riemannian metric is positive, and for general
RLC networks the metric is indefinite), and dissipative systems where the inertial effects are neglected. The class of gradient control systems can be regarded as a counterpart of the class of Hamiltonian control systems as extensively studied in e.g. (Crouch and van der Schaft 1987, van der Schaft 1984b), where the Riemannian metric is replaced by a symplectic structure on the state space manifold.

In (Crouch and van der Schaft 1987, Crouch et al. 1995) necessary and sufficient conditions have been given under which a minimal nonlinear affine control system with an equal number of inputs and outputs is a Hamiltonian control system with respect to some symplectic structure (which then turns out to be unique!). In the present paper 
we describe an analogous theory for the gradient case. All proofs and further elaborations will be contained in (Cortés et al. 2003).

The present paper is organized as follows. In Section 2 we present the class of nonlinear gradient systems. We also introduce the notions of prolongation and gradient extension of a nonlinear system, whose observability properties are studied in Section 3. Section 4 introduces the concept of (weakly) externally equivalent systems. In Section 5 , we introduce the important notion of compatibility between a nonlinear system and a given affine connection. At this point, we are ready to state the main result, namely the characterization of when a general nonlinear control system is a gradient system.

\section{GENERAL SETTING}

Let $M$ be an $n$-dimensional differentiable manifold. We will denote by $T M, T^{*} M$ the tangent and cotangent bundles of $M$, by $\mathfrak{X}(M)$ the set of smooth vector fields on $M$, by $\Omega^{1}(M)$ the set of smooth one-forms on $M$, and by $C^{\infty}(M)$ the set of smooth functions on $M$. Throughout the paper, the manifold $M$ and the mathematical objects defined on it will be assumed to be real-analytic. Consider a nonlinear control system $\Sigma$ with state space $M$, affine in the inputs, and with an equal number of inputs and outputs,

$$
\Sigma:\left\{\begin{array}{l}
\dot{x}=g_{0}(x)+\sum_{j=1}^{m} u_{j} g_{j}(x), \\
y_{j}=V_{j}(x), \quad j=1, \ldots, m,
\end{array}\right.
$$

where $x \in M, x(0)=x_{0}$ and $u=\left(u_{1}, \ldots, u_{m}\right) \in$ $U \subset \mathbb{R}^{m}$. The vector fields $g_{0}, g_{1}, \ldots, g_{m}$ on $M$ are assumed to be complete and $V_{1}, \ldots, V_{m}$ are realvalued functions on $M$. The set $U$ is the control space, which for simplicity is assumed to be an open subset of $\mathbb{R}^{m}$, containing 0 . The function $u(t)=\left(u_{1}(t), \ldots, u_{m}(t)\right)$ belongs to a certain class of functions of time, denoted by $\mathcal{U}$, called the admissible controls. For our purposes, we may restrict the admissible controls to be the piecewise constant right continuous functions.

An important subclass of the family of nonlinear systems (1) is formed by the gradient control systems. Let $\mathcal{G}$ be a pseudo-Riemannian metric on $M$, i.e. a non-degenerate symmetric $(0,2)$-tensor on $M$ (not necessarily positive definite). Consider the 'musical' isomorphisms, $b_{\mathcal{G}}: \mathfrak{X}(M) \rightarrow \Omega^{1}(M)$, $\sharp_{\mathcal{G}}: \Omega^{1}(M) \rightarrow \mathfrak{X}(M)$ defined by

$$
b_{\mathcal{G}}(X)(Y)=\mathcal{G}(X, Y), \quad \sharp_{\mathcal{G}}(\omega)=b_{\mathcal{G}}^{-1}(\omega),
$$

where $X, Y \in \mathfrak{X}(M)$ and $\omega \in \Omega^{1}(M)$. The gradient vector field associated with $V \in C^{\infty}(M)$ is given by $\operatorname{grad}_{\mathcal{G}} V=\sharp_{\mathcal{G}}(d V)$. Reciprocally, $X \in$ $\mathfrak{X}(M)$ is said to be locally gradient if $b_{\mathcal{G}}(X)$ is closed. By Poincaré's lemma, this is equivalent to saying that there exists a locally defined function
$V \in C^{\infty}(M)$ with $b_{\mathcal{G}}(X)=d V$. If this equality holds globally, $X$ is called gradient and will be denoted by $X=\operatorname{grad}_{\mathcal{G}} V$. If we fix coordinates $\left(x^{1}, \ldots, x^{n}\right)$ on $M$, then the pseudo-Riemannian metric can be locally expressed as $\mathcal{G}=\mathcal{G}_{a b} d x^{a} \otimes$ $d x^{b}$, where $\left(\mathcal{G}^{a b}=\mathcal{G}\left(\frac{\partial}{\partial x^{a}}, \frac{\partial}{\partial x^{b}}\right)\right)$ is a symmetric matrix. The musical isomorphisms are then given by $b_{\mathcal{G}}=\mathcal{G}_{a b} d x^{a} \otimes d x^{b}, \sharp_{\mathcal{G}}=\mathcal{G}^{a b} \frac{\partial}{\partial x^{a}} \otimes \frac{\partial}{\partial x^{b}}$, where $\left(\mathcal{G}^{a b}\right)$ is the inverse matrix of $\left(\mathcal{G}_{a b}\right)$. Finally, the gradient vector field associated with $V$ reads $\operatorname{grad}_{\mathcal{G}} V=\mathcal{G}^{a b}\left(\partial V / \partial x^{b}\right) \partial / \partial x^{a}$.

Now, assume that the state space of (1) is a pseudo-Riemannian manifold, $(M, \mathcal{G})$. Assume further that the drift $g_{0}$ is locally gradient, and the inputs $g_{j}$ are gradient with respect to the functions $V_{1}, \ldots, V_{m}$, i.e. $g_{j}=\operatorname{grad}_{\mathcal{G}} V_{j}, j=1, \ldots, m$. Then, the resulting system

$$
\Sigma:\left\{\begin{array}{l}
\dot{x}=g_{0}(x)+\sum_{j=1}^{m} u_{j}(t) \operatorname{grad}_{\mathcal{G}} V_{j}(x), \\
y_{j}=V_{j}(x), \quad j=1, \ldots, m,
\end{array}\right.
$$

is called a locally gradient control system on $M$. If the drift $g_{0}$ is gradient, $g_{0}=\operatorname{grad}_{\mathcal{G}} V_{0}$, then the system is called a gradient control system on $M$. Our objective is to characterize when a nonlinear system of the form (1) is a locally gradient control system (2), i.e. find necessary and sufficient conditions for the existence of a pseudoRiemannian metric on $M$ such that (1) is of the form (2). These conditions are given in terms of the output behavior of the prolongation and the gradient extension of $\Sigma$, which we describe next.

\subsection{The prolongation of a nonlinear system}

This subsection is based on (Crouch and van der Schaft 1987). The prolongation of (1) corresponds to considering together the original system (1) and the (locally defined) variational systems along its state-input-output trajectories. An intrinsic definition of the prolongation on the whole tangent space $T M$ can be stated resorting to the notions of vertical and complete lifts of functions and vector fields (Yano and Ishihara 1973).

Definition 1. The prolongation $\Sigma^{p}$ of a nonlinear system $\Sigma$ of the form (1) is defined by

$$
\begin{gathered}
\dot{x}_{p}=g_{0}^{\mathrm{C}}\left(x_{p}\right)+\sum_{j=1}^{m} u_{j}(t) g_{j}^{\mathrm{C}}\left(x_{p}\right)+\sum_{j=1}^{m} u_{j}^{\mathrm{V}}(t) g_{j}^{\mathrm{V}}\left(x_{p}\right) \\
y_{j}=V_{j}^{\mathrm{V}}\left(x_{p}\right), \quad y_{j}^{\mathrm{V}}=V_{j}^{\mathrm{C}}\left(x_{p}\right), j=1, \ldots, m, \quad(3) \\
\text { where } x_{p}=(x, v) \in T M, \text { and } x_{p}(0)=\left(x_{0}, v_{0}\right) .
\end{gathered}
$$

Remark 2. Analogously, one can introduce the notions of adjoint variational system and Hamiltonian extension. These notions play a key role in the characterization of when a general system admits a Hamiltonian description, see (Crouch and van der Schaft 1987). 


\subsection{The gradient extension of a nonlinear system}

When dealing with the Hamiltonian extension of a nonlinear system, one relies on the fact that the cotangent bundle is endowed with a canonical symplectic structure. However, this is not the case when treating gradient systems, since a canonical pseudo-Riemannian structure on the cotangent bundle does not exist. In order to define the gradient extension of a nonlinear system of the form (1), we will first select a torsion-free affine connection $\nabla$ on $M$, and then consider its Riemannian extension to $T^{*} M$ (cf. (Patterson and Walker 1952)).

Let us briefly present some basic notions on affine connections and Riemannian geometry. An affine connection (Kobayashi and Nomizu 1963) on a manifold $M$ is defined as an assignment

$$
\begin{aligned}
\nabla: \mathfrak{X}(M) \times \mathfrak{X}(M) & \longrightarrow \mathfrak{X}(M) \\
(X, Y) & \longmapsto \nabla_{X} Y
\end{aligned}
$$

which is $\mathbb{R}$-bilinear and satisfies $\nabla_{f X} Y=f \nabla_{X} Y$ and $\nabla_{X}(f Y)=f \nabla_{X} Y+X(f) Y$, for any $X, Y \in$ $\mathfrak{X}(M), f \in C^{\infty}(M)$. Let $c: t \in\left[t_{0}, t_{1}\right] \mapsto c(t)=$ $\left(x^{1}(t), \ldots, x^{n}(t)\right) \in M$ be a curve on $M$ and $W$ a vector field along $c$, i.e. a map $W:\left[t_{0}, t_{1}\right] \rightarrow T M$ such that $\tau_{M}(W(t))=c(t)$ for all $t \in[a, b]$. Let $V$ be a vector field that satisfies $V(c(t))=W(t)$. The covariant derivative of $W$ along $c$ is

$$
\frac{D W(t)}{d t}=\nabla_{\dot{c}(t)} W(t)=\left.\nabla_{\dot{c}(t)} V(x)\right|_{x=c(t)} .
$$

The torsion tensor of an affine connection is defined by $T: \mathfrak{X}(M) \times \mathfrak{X}(M) \rightarrow \mathfrak{X}(M),(X, Y) \mapsto$ $\nabla_{X} Y-\nabla_{Y} X-[X, Y]$. Locally, we have

$$
T\left(\frac{\partial}{\partial x^{a}}, \frac{\partial}{\partial x^{b}}\right)=\left(\Gamma_{a b}^{c}-\Gamma_{b a}^{c}\right) \frac{\partial}{\partial x^{c}},
$$

where the $\Gamma_{b c}^{a}(x)$ are the Christoffel symbols of the affine connection, defined by

$$
\nabla_{\frac{\partial}{\partial x^{b}}} \frac{\partial}{\partial x^{c}}=\Gamma_{b c}^{a}(x) \frac{\partial}{\partial x^{a}} .
$$

Given an affine connection, the symmetric product (Lewis and Murray 1997) of two vector fields $X, Y \in \mathfrak{X}(M)$ is defined by the operation

$$
\langle X: Y\rangle=\nabla_{X} Y+\nabla_{Y} X .
$$

The symmetric product plays a crucial role within the so-called affine connection formalism of mechanical control systems in the study of a variety of aspects such as controllability, series expansions, motion planning and optimal control (Bullo and Lewis 2003).

Associated with the metric $\mathcal{G}$ there is a natural affine connection, called the Levi-Civita connection (Do Carmo 1992). The Levi-Civita connection $\nabla^{\mathcal{G}}$ is determined by the formula

$$
\begin{aligned}
& 2 \mathcal{G}\left(\nabla_{X}^{\mathcal{G}} Y, Z\right) \\
& \quad=X(\mathcal{G}(Y, Z))+Y(\mathcal{G}(Z, X))-Z(\mathcal{G}(X, Y)) \\
& +\mathcal{G}(Y,[Z, X])-\mathcal{G}(X,[Y, Z])+\mathcal{G}(Z,[X, Y])
\end{aligned}
$$

$X, Y, Z \in \mathfrak{X}(M)$, and it is torsion-free, i.e., $T(X, Y)=0$, for any $X, Y \in \mathfrak{X}(M)$. Therefore a pseudo-Riemannian metric on $M$ defines a unique affine connection on $M$, but the converse is not always true. Given a pseudo-Riemannian metric $\mathcal{G}$ on $M$, we can define the Beltrami bracket (Crouch 1981, van der Schaft 1984a),

$$
\{f: g\}_{\mathcal{G}}=\mathcal{G}\left(\operatorname{grad}_{\mathcal{G}} f, \operatorname{grad}_{\mathcal{G}} g\right), f, g \in C^{\infty}(M) \text {. }
$$

In local coordinates, one has the expression,

$$
\{f: g\}_{\mathcal{G}}=\frac{\partial f}{\partial x^{a}} \mathcal{G}^{a b} \frac{\partial g}{\partial x^{b}} .
$$

It is interesting to note that the mapping $\operatorname{grad}_{\mathcal{G}}$ : $\left(C^{\infty}(M),\{\cdot: \cdot\}_{\mathcal{G}}\right) \rightarrow\left(\mathfrak{X}(M),\langle\cdot: \cdot\rangle_{\nabla^{\mathcal{G}}}\right)$ is a homomorphism of symmetric algebras, that is, $\operatorname{grad}_{\mathcal{G}}\{f: g\}_{\mathcal{G}}=\left\langle\operatorname{grad}_{\mathcal{G}} f: \operatorname{grad}_{\mathcal{G}} g\right\rangle_{\nabla_{\mathcal{G}}}$, for all $f, g \in C^{\infty}(M)$.

Let us now turn our discussion to the cotangent bundle of $M$. With each vector field $X$ on $M$ we associate a function $V^{X}$ on $T^{*} M$, defined by $V^{X}(x, p)=\langle p, X(x)\rangle$. The notion of vertical lift of a function $V$ on $M$ to a function $V^{\mathrm{V}}$ on $T^{*} M$ is given by $V^{\mathrm{V}}=V \circ \pi_{M}$, where $\pi_{M}$ is the cotangent bundle projection. An object which plays a key role in the subsequent discussion is the Riemannian extension (Patterson and Walker 1952, Yano and Ishihara 1973) of a torsion-free affine connection. Let $\nabla$ be a torsion-free affine connection on $M$. Then $\nabla$ defines a pseudo-Riemannian metric on $T^{*} M$, denoted $\mathcal{G}^{\mathrm{C}}$, as the unique $(0,2)$ tensor on $T^{*} M$ satisfying $\mathcal{G}^{\mathrm{C}}\left(X^{\mathrm{C}}, Y^{\mathrm{C}}\right)=-V^{\langle X: Y\rangle}$. The matrix representations of the musical isomorphisms defined by $\mathcal{G}^{\mathrm{C}}$ in the induced local coordinates $\left(x^{1}, \ldots, x^{n}, p_{1}, \ldots, p_{n}\right)$ on $T^{*} M$ are given by

$$
b_{\mathcal{G}^{\mathrm{C}}} \equiv\left(\begin{array}{cc}
-2 p_{c} \Gamma_{a b}^{c} & I_{n} \\
I_{n} & 0
\end{array}\right), \quad \sharp_{\mathcal{G}^{\mathrm{C}}} \equiv\left(\begin{array}{cc}
0 & I_{n} \\
I_{n} & 2 p_{c} \Gamma_{a b}^{c}
\end{array}\right) .
$$

As for the gradient vector fields associated with the functions $V^{X}, V^{\mathrm{v}} \in C^{\infty}\left(T^{*} M\right), X \in \mathfrak{X}(M)$, $V \in C^{\infty}(M)$, one has the local expressions

$$
\begin{aligned}
\operatorname{grad}_{\mathcal{G}^{\mathrm{c}}} V^{X} & =X^{a} \frac{\partial}{\partial x^{a}}+p_{a}\left(\frac{\partial X^{a}}{\partial x^{b}}+2 \Gamma_{b c}^{a} X^{c}\right) \frac{\partial}{\partial p_{b}}, \\
\operatorname{grad}_{\mathcal{G}^{\mathrm{C}}} V^{\mathrm{V}} & =\frac{\partial V}{\partial x^{a}} \frac{\partial}{\partial p_{a}} .
\end{aligned}
$$

Definition 3. The gradient extension $\Sigma^{e}$ of a nonlinear system $\Sigma$ of the form (1) with respect to a torsion-free affine connection $\nabla$ on $M$ is given by

$$
\begin{gathered}
\dot{x}_{e}=\operatorname{grad}_{\mathcal{G}^{\mathrm{c}}} V^{g_{0}}\left(x_{e}\right)+\sum_{j=1}^{m} u_{j}(t) \operatorname{grad}_{\mathcal{G}^{\mathrm{c}}} V^{g_{j}}\left(x_{e}\right) \\
+\sum_{j=1}^{m} u_{j}^{a}(t) \operatorname{grad}_{\mathcal{G}^{\mathrm{c}}} V_{j}^{\mathrm{V}}\left(x_{e}\right), \quad \text { (4) } \\
y_{j}=V_{j}^{\mathrm{V}}\left(x_{e}\right), \quad y_{j}^{a}=V^{g_{j}}\left(x_{e}\right), j=1, \ldots, m, \\
\text { with } x_{e}=(x, p) \in T^{*} M, x_{e}(0)=\left(x_{0}, p_{0}\right), \\
\left(u_{1}, \ldots, u_{m}\right) \in U \subset \mathbb{R}^{m},\left(u_{1}^{a}, \ldots, u_{m}^{a}\right) \in \mathbb{R}^{m} .
\end{gathered}
$$

Remark 4. Note that the gradient extension $\Sigma^{e}$ is itself a gradient control system. 


\section{OBSERVABILITY OF THE PROLONGATION AND THE GRADIENT EXTENSION}

In this section, we investigate the observability properties of the prolonged system and the gradient extension of a nonlinear system. We start by briefly reviewing some notions such as distinguishable points and local observability.

Let $\mathcal{Y}$ denote the space of absolutely continuous functions on $M$ with values in $\mathbb{R}^{m}$. Let $\mathcal{R}_{\Sigma}: M \times$ $\mathcal{U} \rightarrow \mathcal{Y}, \mathcal{R}_{\Sigma}\left(x_{0}, u(t)\right)=\left(V_{1}\left(x\left(t, x_{0}, u(t)\right)\right), \ldots\right.$, $\left.V_{m}\left(x\left(t, x_{0}, u(t)\right)\right)\right)$ denote the input-output map of the nonlinear system (1). Two points $x_{1}, x_{2} \in$ $M$ are said to be indistinguishable, $x_{1} \sim x_{2}$, if $\mathcal{R}_{\Sigma}\left(x_{1}, u(\cdot)\right)=\mathcal{R}_{\Sigma}\left(x_{2}, u(\cdot)\right)$ for any $u(\cdot) \in \mathcal{U}$.

Definition 5. A system $\Sigma$ is observable if for any $x_{1}, x_{2} \in M$, one has that $x_{1} \sim x_{2} \Rightarrow x_{1}=x_{2}$. Alternatively, for any $x_{1} \neq x_{2}$, there exists an admissible control such that the output functions resulting from the initial conditions $x(0)=x_{1}$, resp. $x(0)=x_{2}$, are different. The system is locally observable at $x_{0}$ if there exists a neighborhood $\mathcal{N}$ of $x_{0}$ such that this holds for points in $\mathcal{N}$.

Denote by $\mathcal{H}$ the $\mathbb{R}$-linear space in $C^{\infty}(M)$ spanned by the functions of the form $\mathcal{L}_{X_{1}} \mathcal{L}_{X_{2}} \ldots$ $\mathcal{L}_{X_{s}} V_{j}$, with $\left\{X_{r}\right\}_{r=1}^{s} \subset\left\{g_{i} \mid i=0,1, \ldots, m\right\}$, and $j \in\{1, \ldots, m\}$. Alternatively, we may take $X_{r}$ to be arbitrary elements of the accessibility algebra corresponding to the vector fields $g_{0}, g_{1}, \ldots, g_{m} . \mathcal{H}$ is called the observation space of $\Sigma$. It follows from the analyticity assumption that the system is observable if and only if $\mathcal{H}$ distinguishes points in $M$, i.e. for every $x_{1}, x_{2} \in M$ with $x_{1} \neq x_{2}$, there exists $V \in \mathcal{H}$ such that $V\left(x_{1}\right) \neq V\left(x_{2}\right)$, cf. (Hermann and Krener 1977).

Proposition 6. ((Crouch and van der Schaft 1987)). Consider a nonlinear system $\Sigma$ of the form (1), with observation space $\mathcal{H}$. Then, the observation space $\mathcal{H}^{p}$ of the prolongation $\Sigma^{p}$ is given by $\mathcal{H}^{p}=\mathcal{H}^{\mathrm{C}}+\mathcal{H}^{\mathrm{V}}$, where $\mathcal{H}^{\mathrm{C}}=\left\{V^{\mathrm{C}} \mid V \in \mathcal{H}\right\}$ and $\mathcal{H}^{\mathrm{v}}=\left\{V^{\mathrm{v}} \mid V \in \mathcal{H}\right\}$.

The following corollary is a modified statement of Corollary 3.3 in (Crouch and van der Schaft 1987).

Corollary 7. Assume the codistribution $d \mathcal{H}$ is of constant rank. Then the system $\Sigma$ is (locally) observable if and only if its prolongation is (locally) observable.

Let us turn our attention to the observability properties of the gradient extension of a nonlinear system of the form (1). The following lemma will be most helpful.

Lemma 8. Let $\nabla$ be a torsion-free affine connection on a manifold $M$, and let $\mathcal{G}^{\mathrm{C}}$ denote its
Riemannian extension to $T^{*} M$. Then, for any vector fields $X, Y \in \mathfrak{X}(M)$, and any functions $f, g \in C^{\infty}(M)$, the following identities hold

(i) $\left\{V^{X}: V^{Y}\right\}_{\mathcal{G}^{\mathrm{c}}}=V^{\langle X: Y\rangle}=-\mathcal{G}^{\mathrm{C}}\left(X^{\mathrm{C}}, Y^{\mathrm{C}}\right)$.

(ii) $\left(\operatorname{grad}_{\mathcal{G}^{\mathrm{c}}} V^{X}\right)\left(f^{\mathrm{v}}\right)=\left(\operatorname{grad}_{\mathcal{G}^{\mathrm{c}}} f^{\mathrm{v}}\right)\left(V^{X}\right)=\left\{V^{X}\right.$ : $\left.f^{\mathrm{V}}\right\}_{\mathcal{G}^{\mathrm{C}}}=X(f)^{\mathrm{V}}$.

(iii) $\left(\operatorname{grad}_{\mathcal{G}^{\mathrm{c}}} f^{\mathrm{V}}\right)\left(g^{\mathrm{V}}\right)=\left\{f^{\mathrm{V}}: g^{\mathrm{V}}\right\}_{\mathcal{G}^{\mathrm{c}}}=0$.

Denote by $S_{0}$ the $\mathbb{R}$-linear space in $\mathfrak{X}(M)$ spanned by the vector fields of the form $\left\langle X_{1}:\left\langle X_{2}\right.\right.$ : $\left.\left.\left\langle\ldots:\left\langle X_{s}: g_{j}\right\rangle\right\rangle \ldots\right\rangle\right\rangle$, with $\left\{X_{r}\right\}_{r=1}^{s} \subset\left\{g_{i} \mid i=\right.$ $0,1, \ldots, m\}$, and $j \in\{1, \ldots, m\}$. Alternatively, one can define $S_{0}$ as the smallest subspace of $\mathfrak{X}(M)$ such that (i) $g_{1}, \ldots, g_{m} \in S_{0}$; and (ii) if $X \in S_{0}$, then $\left\langle g_{i}: X\right\rangle \in S_{0}$ for all $i=0,1, \ldots, m$. We denote by $\mathcal{S}_{0}$ the distribution on $M$ generated by the space $S_{0}$,

$$
\mathcal{S}_{0}(x)=\operatorname{span}\left\{X(x) \mid X \in S_{0}\right\}, \quad x \in M .
$$

Proposition 9. Consider a nonlinear system $\Sigma$ of the form (1), with observation space $\mathcal{H}$. Let $\nabla$ be a torsion-free affine connection on $M$. Then, the observation space $\mathcal{H}^{e}$ of the gradient extension $\Sigma^{e}$ is given by $\mathcal{H}^{e}=V^{\mathcal{S}_{0}}+(\mathcal{H}+\mathfrak{h})^{\mathrm{v}}$, where $V^{\mathcal{S}_{0}}=\left\{V^{X} \mid X \in \mathcal{S}_{0}\right\}$ and $\mathfrak{h}$ is spanned by $\mathcal{L}_{X_{1}} \mathcal{L}_{X_{2}} \ldots \mathcal{L}_{X_{s}} \mathcal{L}_{X} V_{j}$, with $X_{r}, r=1, \ldots, s$, equal to $g_{i}, i=0,1, \ldots, m, X \in \mathcal{S}_{0}$.

Proof: The observation space of the gradient extension of $\Sigma$ is spanned by

$$
\mathcal{L}_{X_{1}} \mathcal{L}_{X_{2}} \ldots \mathcal{L}_{X_{s}} V_{j}^{\mathrm{V}}, \quad \mathcal{L}_{X_{1}} \mathcal{L}_{X_{2}} \ldots \mathcal{L}_{X_{s}} V^{g_{j}},
$$

where $X_{r}, r=1, \ldots, s$ is equal to $\operatorname{grad}_{\mathcal{G}^{c}} V^{g_{i}}$, $\operatorname{grad}_{\mathcal{G}^{\mathrm{c}}} V_{j}^{\mathrm{V}}, i=0,1, \ldots, m, j=1, \ldots, m$. Now, using Lemma 8, we have

$$
\begin{array}{ll}
\mathcal{L}_{\operatorname{grad}_{\mathcal{G}^{\mathrm{C}}} V^{g_{i}} V_{j}^{\mathrm{V}}=\left(\mathcal{L}_{g_{i}} V_{j}\right)^{\mathrm{V}},}, \mathcal{L}_{\operatorname{grad}_{\mathcal{G}^{\mathrm{C}}} V^{g_{i}} V^{g_{j}}=V^{\left\langle g_{i}: g_{j}\right\rangle},}, \\
\mathcal{L}_{\operatorname{grad}_{\mathcal{G}^{\mathrm{C}}} V_{j}^{\mathrm{v}}} V_{k}^{\mathrm{V}}=0, & \mathcal{L}_{\operatorname{grad}_{\mathcal{G}^{\mathrm{C}}} V_{j}^{\mathrm{v}}} V^{g_{k}}=\left(\mathcal{L}_{g_{k}} V_{j}\right)^{\mathrm{V}},
\end{array}
$$

with $i=0,1, \ldots, m$ and $j, k=1, \ldots, m$. Considering the next step of Lie derivatives yields

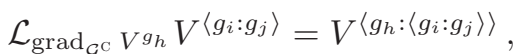

$$
\begin{aligned}
& \mathcal{L}_{\operatorname{grad}_{\mathcal{G}^{\mathrm{C}}} V^{g_{h}}}\left(\mathcal{L}_{g_{i}} V_{j}\right)^{\mathrm{V}}=\left(\mathcal{L}_{g_{h}} \mathcal{L}_{g_{i}} V_{j}\right)^{\mathrm{V}}, \\
& \mathcal{L}_{\operatorname{grad}_{\mathcal{G}^{\mathrm{C}}} V_{k}^{\mathrm{v}}} V^{\left\langle g_{i}: g_{j}\right\rangle}=\left(\mathcal{L}_{\left\langle g_{i}: g_{j}\right\rangle} V_{k}\right)^{\mathrm{V}}, \\
& \mathcal{L}_{\operatorname{grad}_{\mathcal{G}^{\mathrm{C}}} V_{k}^{\mathrm{v}}}\left(\mathcal{L}_{g_{i}} V_{j}\right)^{\mathrm{V}}=0,
\end{aligned}
$$

with $h=0,1, \ldots, m$. Further iterating this process, we get to the desired result.

Corollary 10. Consider a nonlinear system $\Sigma$ of the form (1), with observation space $\mathcal{H}$. Assume the codistribution $d \mathcal{H}$ is of constant rank. Let $\nabla$ be a torsion-free affine connection on $M$ and further assume that the distribution $\mathcal{S}_{0}$ is fullrank. Then, $\Sigma$ is (locally) observable implies that $\Sigma^{e}$ is (locally) observable.

Proof: Since the codistribution $d \mathcal{H}$ has constant rank, $\Sigma$ is locally observable if and only if $\operatorname{dim} d \mathcal{H}(x)=\operatorname{dim} M$. Since $\mathcal{S}_{0}$ is full-rank, it is 
clear that $\Sigma$ locally observable implies that $\mathcal{H}^{e}$ has constant maximal rank, and therefore $\Sigma^{e}$ is locally observable. With respect to observability, let $\left(x_{1}, p_{1}\right),\left(x_{2}, p_{2}\right) \in T^{*} M$ and assume that $V^{e}\left(x_{1}, p_{1}\right)=V^{e}\left(x_{2}, p_{2}\right)$ for all $V^{e} \in \mathcal{H}^{e}$. Since $\mathcal{H}^{\mathrm{V}} \subset \mathcal{H}^{e}$, this yields $V\left(x_{1}\right)=V\left(x_{2}\right)$ for any $V \in \mathcal{H}$. So, under observability of $\Sigma$, we conclude that $x_{1}=x_{2}=x$. Then, we have that $V^{X}\left(x, p_{1}\right)=V^{X}\left(x, p_{2}\right)$, for all $X \in S_{0}$, which finally implies that $p_{1}=p_{2}$.

\section{EXTERNALLY EQUIVALENT SYSTEMS}

In this section we introduce the notion of (weakly) externally equivalent systems, which will be instrumental in the statement of the main result in Section 5. Consider two nonlinear systems $\Sigma^{\alpha}$, $\alpha=1,2$ of the form,

$$
\begin{aligned}
& \dot{x}^{\alpha}=g_{0}^{\alpha}\left(x^{\alpha}\right)+\sum_{j=1}^{m} u_{j} g_{j}^{\alpha}\left(x^{\alpha}\right), \quad x^{\alpha} \in M^{\alpha}, \\
& y_{j}=V_{j}^{\alpha}\left(x^{\alpha}\right), u=\left(u_{1}, \ldots, u_{m}\right) \in U \subset \mathbb{R}^{m} .
\end{aligned}
$$

Denote by $\mathcal{H}^{\alpha}, \alpha=1,2$, the associated observation spaces. Take a function $H^{1} \in \mathcal{H}^{1}, H^{1}=$ $\mathcal{L}_{X_{1}} \ldots \mathcal{L}_{X_{s}} V_{j}^{1}$, with $X_{r}=g_{i_{r}}^{1}, i_{r} \in\{0,1, \ldots, m\}$, $r=1, \ldots, s$ and $j \in\{1, \ldots, m\}$. Consider the function in $\mathcal{H}^{2}$ defined by $H^{2}=\mathcal{L}_{Y_{1}} \ldots \mathcal{L}_{Y_{s}} V_{j}^{2}$, with $Y_{r}=g_{i_{r}}^{2}, r=1, \ldots, s$. Then we say that $H^{1}$ and $H^{2}$ formally correspond to each other. This notion is useful to define the concept of weakly externally equivalent systems.

Definition 11 . The systems $\Sigma^{1}$ and $\Sigma^{2}$ are weakly externally equivalent if and only if for all $x^{1} \in M^{1}$, there exists $x^{2} \in M^{2}$ such that $H^{1}\left(x^{1}\right)=H^{2}\left(x^{2}\right)$ for all corresponding $H^{1} \in \mathcal{H}^{1}, H^{2} \in \mathcal{H}^{2}$, and vice versa.

Definition 12. The systems $\Sigma^{1}$ and $\Sigma^{2}$ are externally equivalent if and only if for all $x^{1} \in M^{1}$, there exists $x^{2} \in M^{2}$ such that the input-output maps corresponding to $x^{1}$ and $x^{2}$ coincide, i.e. $\mathcal{R}_{\Sigma^{1}}\left(x^{1}, u(\cdot)\right)=\mathcal{R}_{\Sigma^{2}}\left(x^{2}, u(\cdot)\right)$, for all $u(\cdot) \in \mathcal{U}$, and vice versa.

Equivalently, $\Sigma^{1}$ and $\Sigma^{2}$ are externally equivalent if and only if their behaviors are equal. Clearly, if two systems are externally equivalent, then they are weakly externally equivalent.

Proposition 13. Assume that $\Sigma^{1}$ and $\Sigma^{2}$ are weakly externally equivalent, observable and that the codistributions $d \mathcal{H}^{\alpha}, \alpha=1,2$, have constant rank. Then there exists a unique diffeomorphism $\varphi: M^{1} \rightarrow M^{2}$ with $\varphi^{*}\left(\mathcal{H}^{2}\right)=\mathcal{H}^{1}$.

Corollary 14. Let the systems $\Sigma^{1}$ and $\Sigma^{2}$ be observable and the codistributions $d \mathcal{H}^{\alpha}, \alpha=1,2$, have constant rank. Then $\Sigma^{1}$ and $\Sigma^{2}$ are weakly externally equivalent if and only if they are externally equivalent.

\section{GRADIENT REALIZATION OF A NONLINEAR CONTROL SYSTEM}

This section contains the main result of the paper. Under certain technical conditions, Theorem 17 below characterizes when a nonlinear control systems admits a gradient realization. Before stating this result, we need to introduce the novel notion of compatibility between a nonlinear system and an affine connection.

Definition 15. Let $\nabla$ be an affine connection on $M$. A nonlinear control system $\Sigma$ of the form (1) is compatible with $\nabla$ if and only if the following two conditions hold:

(a) For all vector fields $X_{1}, \ldots, X_{s_{1}}, Y_{1}, \ldots, Y_{s_{2}} \in$ $\left\{g_{0}, g_{1}, \ldots, g_{m}\right\}$, and all indexes $j, k=1, \ldots, m$,

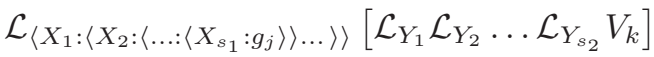

$$
\begin{aligned}
& =\mathcal{L}_{\left\langle Y_{1}:\left\langle Y_{2}:\left\langle\ldots:\left\langle Y_{s_{2}}: g_{k}\right\rangle\right\rangle \ldots\right\rangle\right\rangle}\left[\mathcal{L}_{X_{1}} \mathcal{L}_{X_{2}} \ldots \mathcal{L}_{X_{s_{1}}} V_{j}\right] .
\end{aligned}
$$

(b) For all vector fields $X_{1}, \ldots, X_{s_{1}}, Y_{1}, \ldots, Y_{s_{2}}$, $Z_{1}, \ldots, Z_{s_{3}} \in\left\{g_{0}, g_{1}, \ldots, g_{m}\right\}$, and all indexes $j, k, l=1, \ldots, m$,

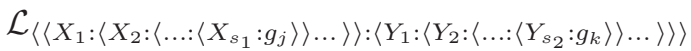

$$
\begin{aligned}
& {\left[\mathcal{L}_{Z_{1}} \mathcal{L}_{Z_{2}} \ldots \mathcal{L}_{Z_{s_{3}}} V_{l}\right]} \\
& =\mathcal{L}_{\left.\left\langle Z_{1}:\left\langle Z_{2}:\left\langle\ldots:\left\langle Z_{s_{3}}: g_{l}\right\rangle\right\rangle\right\rangle\right\rangle\right\rangle}
\end{aligned}
$$

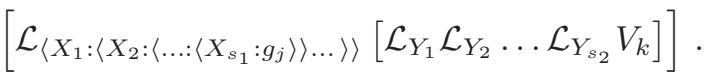

Remark 16. It can be seen (Cortés et al. 2003) that a locally gradient control system of the form (2) is compatible in the above sense with the Levi-Civita connection associated with the pseudo-Riemannian metric $\mathcal{G}$.

Theorem 17. Let $\Sigma$ be a nonlinear control system of the form (1). Let $\nabla$ be a torsion-free affine connection defined on the state manifold $M$. Assume $\Sigma$ is observable with $\operatorname{dim} d \mathcal{H}$ constant, compatible with $\nabla$ and that the distribution $\mathcal{S}_{0}$ is full-rank. Then, $\Sigma$ is a locally gradient control system if and only if its prolonged system $\Sigma^{p}$ and its gradient extension $\Sigma^{e}$ are weakly externally equivalent.

Proof: We only prove the "easy" $\Rightarrow$ direction. For the $\Leftarrow$ direction we refer to the full paper (Cortés et al. 2003). Consider a locally gradient control system $\Sigma$ on $(M, \mathcal{G})$ (cf. (2)), together with its prolongation $\Sigma^{p}$ on $T M$ and its gradient extension $\Sigma^{e}$ on $T^{*} M$. We are going to show that $b_{\mathcal{G}}$ is an isomorphism between the prolongation and the gradient extension, i.e. $b_{\mathcal{G}}\left(x_{p}(\cdot)\right)=x_{e}(\cdot)$ along the solutions of (3) and (4) respectively. This is a consequence of the equalities

$$
\begin{aligned}
\left(b_{\mathcal{G}}\right)_{*} g_{i}^{\mathrm{C}}=\operatorname{grad}_{\mathcal{G}^{\mathrm{c}}} V^{g_{i}} \circ b_{\mathcal{G}}, & & V^{g_{j}} \circ b_{\mathcal{G}}=V_{j}^{\mathrm{C}}, \\
\left(b_{\mathcal{G}}\right)_{*} g_{j}^{\mathrm{V}}=\operatorname{grad}_{\mathcal{G}^{\mathrm{C}}} V_{j}^{\mathrm{V}} \circ b_{\mathcal{G}}, & & V_{j}^{\mathrm{V}} \circ b_{\mathcal{G}}=V_{j}^{\mathrm{V}},
\end{aligned}
$$

for all $i=0,1, \ldots, m, j=1, \ldots, m$. In order to prove these equalities, we make use of the following identities, 


$$
\begin{aligned}
& \left(b_{\mathcal{G}}\right)_{*}\left(\frac{\partial}{\partial x^{a}}\right)=\frac{\partial}{\partial x^{a}}+\frac{\partial \mathcal{G}_{c b}}{\partial x^{a}} v^{b} \frac{\partial}{\partial p_{c}}, \\
& \left(b_{\mathcal{G}}\right)_{*}\left(\frac{\partial}{\partial v^{a}}\right)=\mathcal{G}_{a b} \frac{\partial}{\partial p_{b}} .
\end{aligned}
$$

Let $g \in \mathfrak{X}(M)$. In local coordinates, $g=g^{a} \partial / \partial x^{a}$. Using the definition of complete-lift, we get

$\left(b_{\mathcal{G}}\right)_{*}\left(g^{\mathrm{C}}\right)=g^{a} \frac{\partial}{\partial x^{a}}+\left\{g^{c} \frac{\partial \mathcal{G}_{a b}}{\partial x^{c}}+\mathcal{G}_{a c} \frac{\partial g^{c}}{\partial x^{b}}\right\} v^{b} \frac{\partial}{\partial p_{a}}$.

On the other hand, we have

$$
\begin{aligned}
& \operatorname{grad}_{\mathcal{G}^{\mathrm{C}}} V^{g} \circ b_{\mathcal{G}} \\
& \quad=g^{a} \frac{\partial}{\partial x^{a}}+\left\{\mathcal{G}_{b c} \frac{\partial g^{c}}{\partial x^{a}}+2 \mathcal{G}_{b c} \Gamma_{a d}^{c} g^{d}\right\} v^{b} \frac{\partial}{\partial p_{a}} .
\end{aligned}
$$

Now, suppose that $g$ is a locally gradient vector field. In local coordinates, this means that $\mathcal{G}_{a c} g^{c}=$ $\partial V / \partial x^{a}$, for a certain function $V$, which in turn implies $\partial\left\{\mathcal{G}_{a c} g^{c}\right\} / \partial x^{b}=\partial\left\{\mathcal{G}_{b c} g^{c}\right\} / \partial x^{a}$, that is

$$
\mathcal{G}_{a c} \frac{\partial g^{c}}{\partial x^{b}}=\frac{\partial \mathcal{G}_{b c}}{\partial x^{a}} g^{c}+\mathcal{G}_{b c} \frac{\partial g^{c}}{\partial x^{a}}-\frac{\partial \mathcal{G}_{a c}}{\partial x^{b}} g^{c} .
$$

Substituting into the above expression for $\left(b_{\mathcal{G}}\right)_{*}\left(g^{\mathrm{C}}\right)$, one can derive the equality $\left(b_{\mathcal{G}}\right)_{*} g_{i}^{\mathrm{C}}=\operatorname{grad}_{\mathcal{G}^{\mathrm{C}}} V^{g_{i}} \circ$ $b_{\mathcal{G}}$ for $i=0,1, \ldots, m$. The equality $\left(b_{\mathcal{G}}\right)_{*} g_{j}^{\mathrm{V}}=$ $\operatorname{grad} V_{j}^{\mathrm{V}} \circ b_{\mathcal{G}}, j=1, \ldots, m$, follows by considering the definition of vertical lift and the fact that $g_{j}$ are gradient by hypothesis,

$$
\begin{aligned}
\left(b_{\mathcal{G}}\right)_{*}\left(g_{j}^{\mathrm{V}}\right)=\mathcal{G}_{a b} g_{j}^{b} \frac{\partial}{\partial p_{a}} \\
=\frac{\partial V_{j}}{\partial x^{a}} \frac{\partial}{\partial p_{a}}=\operatorname{grad}_{\mathcal{G}^{\mathrm{c}}} V_{j}^{\mathrm{V}} \circ b_{\mathcal{G}} .
\end{aligned}
$$

As for $V^{g_{j}} \circ b_{\mathcal{G}}=V_{j}^{\mathrm{C}}$, for each $v \in T_{x} M$, we compute $V^{g_{j}} \circ b_{\mathcal{G}}(v)=\mathcal{G}_{a b} v^{b} g_{j}^{a}=\partial V_{j} / \partial x^{b}$. $v^{b}=<d V_{j}, v>=V_{j}^{\mathrm{C}}(v)$. The last equality follows trivially. Consequently, the prolongation and the gradient extension of a nonlinear system $\Sigma$ which is itself gradient are externally equivalent, in particular weakly externally equivalent systems.

Remark 18. In general, we cannot ensure that the drift vector field $g_{0}$ is globally gradient, unless we impose some additional conditions on the topology of the state space $M$ (for instance, that the first Betti number of $M$ is zero). This is analogous to the situation in the Hamiltonian setting (Crouch and van der Schaft 1987).

\section{CONCLUSIONS}

We have discussed necessary and sufficient conditions for a nonlinear control system to be realizable as a gradient control system with respect to a pseudo-Riemannian metric. The results rely on a suitable notion of compatibility of the system with respect to a given affine connection, and on the input-output behavior of the prolonged system and the gradient extension. The symmetric product associated with an affine connection plays a key role in the discussion. We believe that the developments in this paper do not only give insight in the system-theoretic properties of the physically motivated class of gradient control systems, but also shed light on the differentialgeometric properties of gradient and Lagrangian control systems.

\section{ACKNOWLEDGEMENTS}

The first author's work was partially supported by NSF grant CMS-0100162 and by the European Union Training and Mobility of Researchers Program, ERB FMRXCT-970137.

\section{REFERENCES}

Bullo, F. and A. D. Lewis (2003). Geometric Control of Simple Mechanical Systems. Preprint.

Cortés, J., A. J. van der Schaft and P. E. Crouch (2003). Characterization of gradient control systems. Preprint.

Crouch, P. E. (1981). Geometric structures in systems theory. IEE Proceedings 128(5), 242252.

Crouch, P. E. and A. J. van der Schaft (1987). Variational and Hamiltonian Control Systems. Vol. 101 of Lecture Notes in Control and Information Sciences. Springer Verlag. New York, NY.

Crouch, P. E., F. Lamnabhi-Lagarrigue and A. J. van der Schaft (1995). Adjoint and Hamiltonian input-output differential equations. IEEE Transactions on Automatic Control 40, 603-615.

Do Carmo, M. P. (1992). Riemannian Geometry. Birkhäuser. Boston, MA.

Hermann, R. and A. J. Krener (1977). Nonlinear controllability and observability. IEEE Transactions on Automatic Control 22, 728-740.

Kobayashi, S. and K. Nomizu (1963). Foundations of Differential Geometry. Vol. I. Vol. 15 of Interscience Tracts in Pure and Applied Mathematics. Interscience Publishers. New York, NY.

Lewis, A. D. and R. M. Murray (1997). Configuration controllability of simple mechanical control systems. SIAM Journal on Control and Optimization 35(3), 766-790.

Patterson, E. M. and A. G. Walker (1952). Riemann extensions. Quart. J. Math. 3, 19-28.

van der Schaft, A. J. (1984a). Linearization of Hamiltonian and gradient systems. IMA Journal of Mathematical Control \& Information 1, 185-198.

van der Schaft, A. J. (1984b). System Theoretic Descriptions of Physical Systems. Vol. 3 of CWI Tract. Centre for Mathematics and Computer Science. Amsterdam.

Yano, K. and S. Ishihara (1973). Tangent and cotangent bundles. Marcel Dekker. New York. 